\title{
Sublayer Stable Fe Dopant in Porous Pd Metallene Boost Oxygen Reduction Reaction
}

Shaoda Huang, $\uparrow$ Shuanglong Lu, $\dagger^{*}$ Shun Gong, $\ddagger$ Qiuju Zhang, $\ddagger^{*}$ Fang Duan, $\uparrow$ Han Zhu, $\uparrow$ Hongwei Gu, § Weifu Dong, $\uparrow$ Mingliang Du, $\uparrow^{*}$

$\uparrow$ Key Laboratory of Synthetic and Biological Colloids, Ministry of Education, School of Chemical and Material Engineering, Jiangnan University, Wuxi, Jiangsu 214122, P. R. China.

\$ Ningbo Institute of Materials Technology and Engineering Chinese Academy of Sciences, Ningbo, Zhejiang 315201, P.R. China.

$\S$ Key Laboratory of Organic Synthesis of Jiangsu Province, College of Chemistry, Chemical Engineering and Materials Science, Collaborative Innovation Centre of Suzhou Nano Science and Technology, Soochow University,

Suzhou, Jiangsu 215123, P. R. China. 


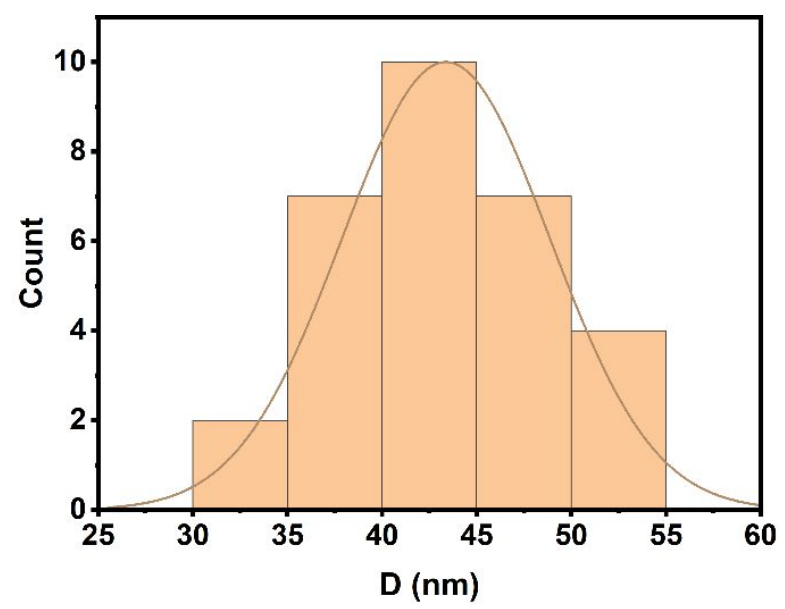

Figure S1. The lateral size distribution of Fe-Pd UPM.

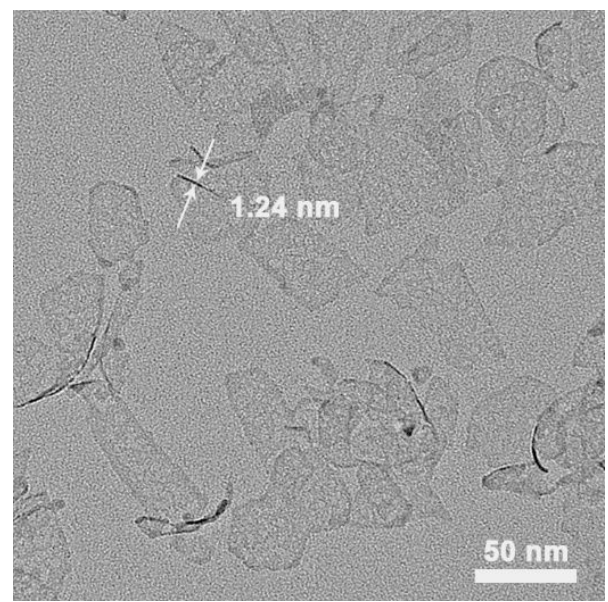

Figure S2. The TEM image of Fe-Pd UPM.

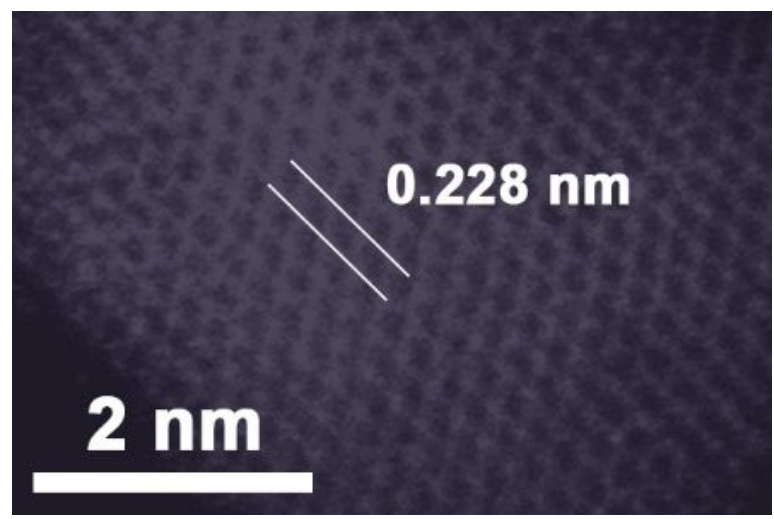

Figure S3. The High-resolution HAADF-STEM image of single Fe-Pd UPM. 

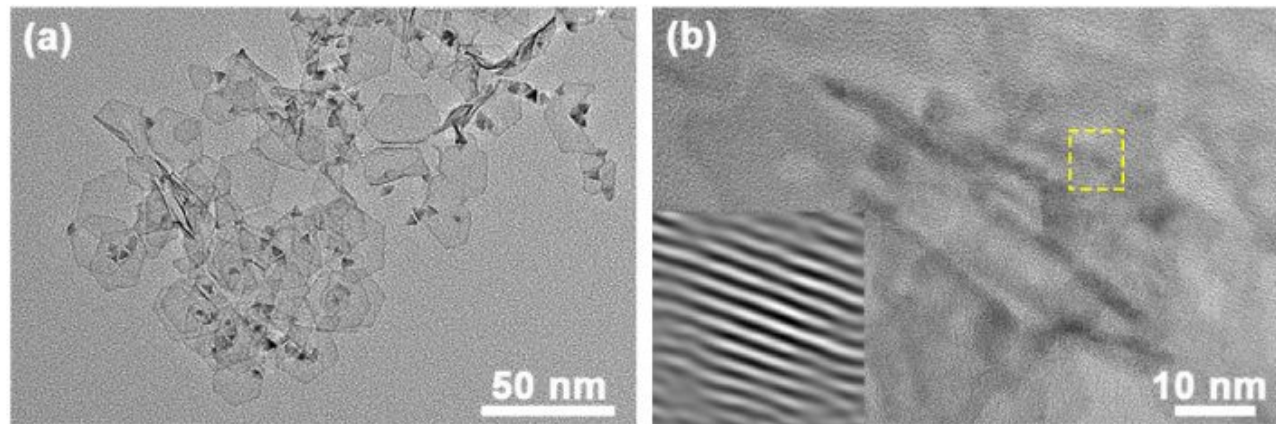

Figure S4. (a) The TEM image and (b) HRTEM H of Pd metallene.

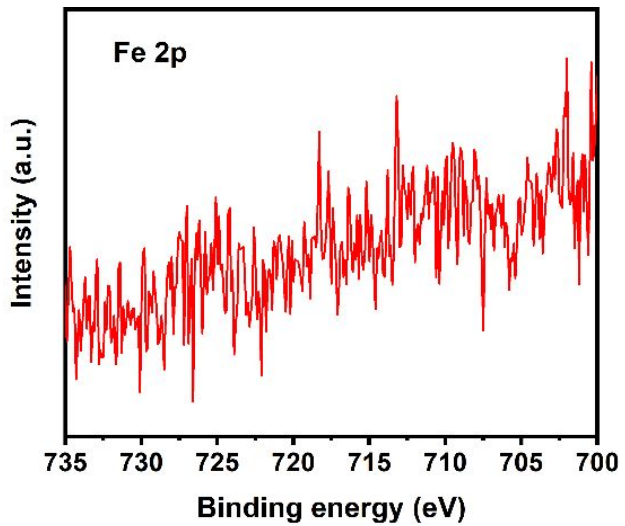

Figure S5. The XPS spectrum of Fe $2 p$ on the Fe-Pd UPM.

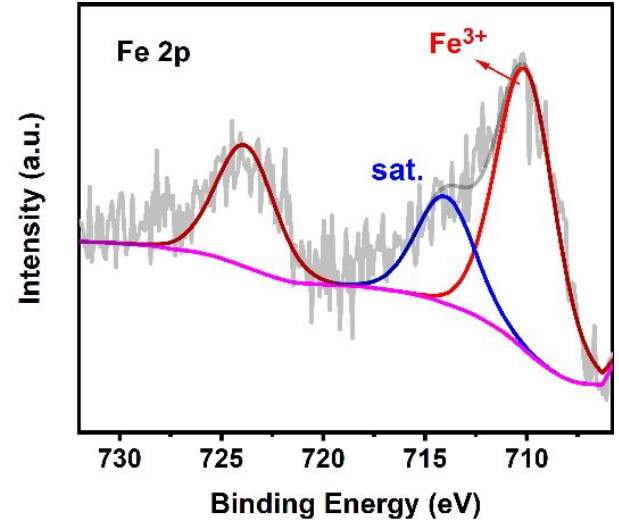

Figure S6. The Fe $2 p$ peaks of Fe-Pd UPM before acid treatment. 
(a)

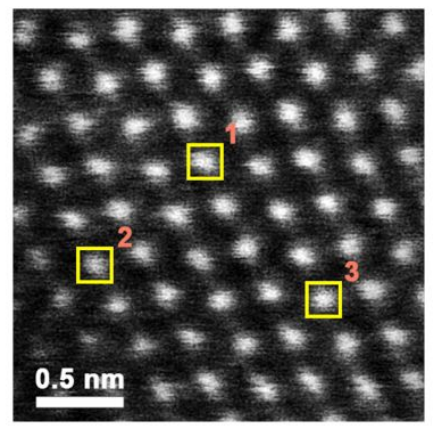

(b)

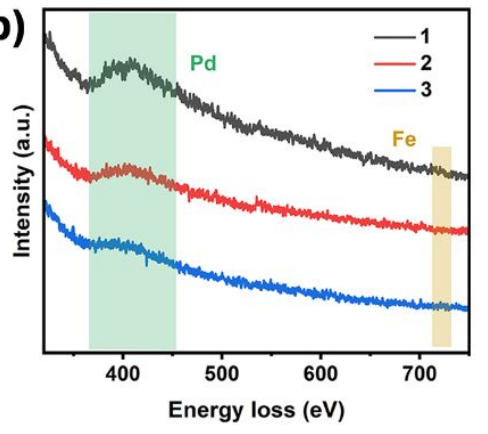

Figure S7: (a) Typical atomic-resolution HAADF-STEM image and (b) corresponding EELS spectrum at the circled areas in (a).

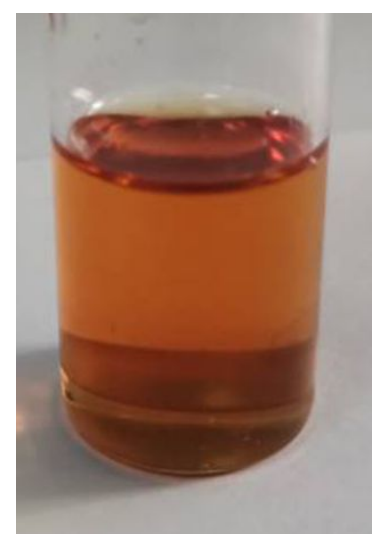

Figure S8. The Photograph of final solution under the same reaction condition as for Fe-Pd UPM but in the absence of $\mathrm{Fe}_{2}(\mathrm{CO})_{9}$
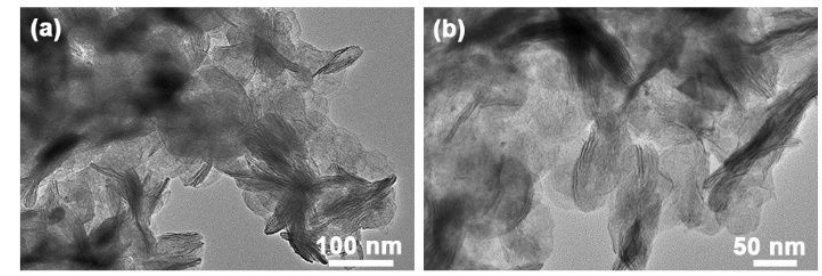

Figure S9. The TEM images of the products under the same reaction condition as for Fe-Pd UPM but in the purged- $\mathrm{N}_{2}$ solution. 

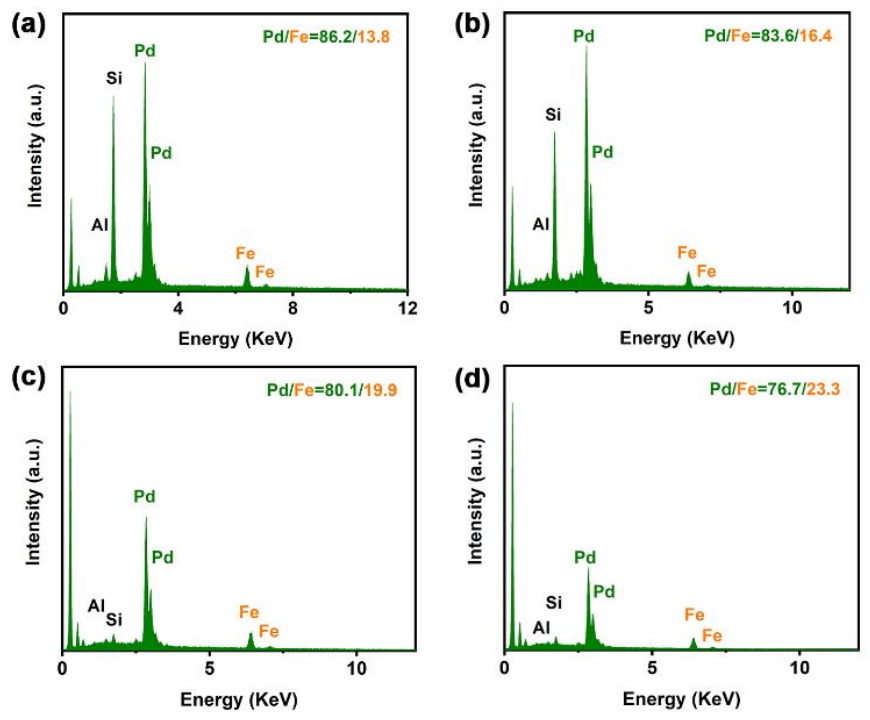

Figure S10. The EDX spectrum of the collected products at different time ((a) $1 \mathrm{~h}$, (b) $2 \mathrm{~h},(\mathrm{c}) 4 \mathrm{~h},(\mathrm{~d})$ $6 h)$.
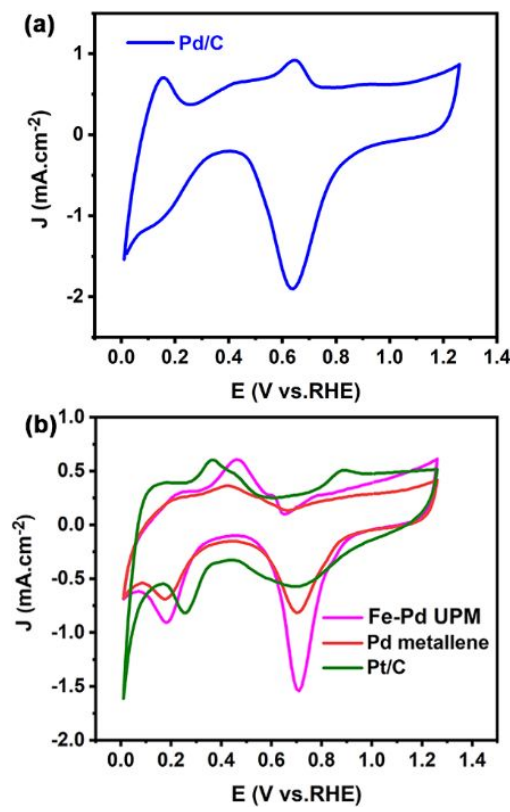

Figure S11. The typical CV curve of commercial Pd/C in $0.1 \mathrm{M} \mathrm{KOH}$ solution at the scan rate of 50 $\mathrm{mV} \mathrm{s}^{-1}$. As shown in Figure $4 \mathrm{a}$ and Figure S11, the several anodic and cathodic waves were observed on the $\mathrm{CV}$ plots, which are similar with previous report. ${ }^{47-49}$ The wave centered at around $0 \mathrm{~V}$ is related to the $\mathrm{H}$ absorption and evolution and the small anodic wave centered at around $0.24 \mathrm{~V}$ are assigned to oxidation of absorbed hydrogen. The two anodic peaks centered at between $0.33^{\sim} 0.64 \mathrm{~V}$ correspond to oxidation of adsorbed hydrogen and the cathodic wave centered at around $0.18 \mathrm{~V}$ correspond to $\mathrm{H}$ adsorption. In addition, the anodic waves centered at above 0.75 $\mathrm{V}$ and the cathodic wave centered at around $0.71 \mathrm{~V}$ could be ascribed to the formation and reduction of surface $\mathrm{Pd}$ oxide. It is noted that the position, shape and size of these peaks show different characteristics on different materials, which probably is due to the electronic and morphology effect. 

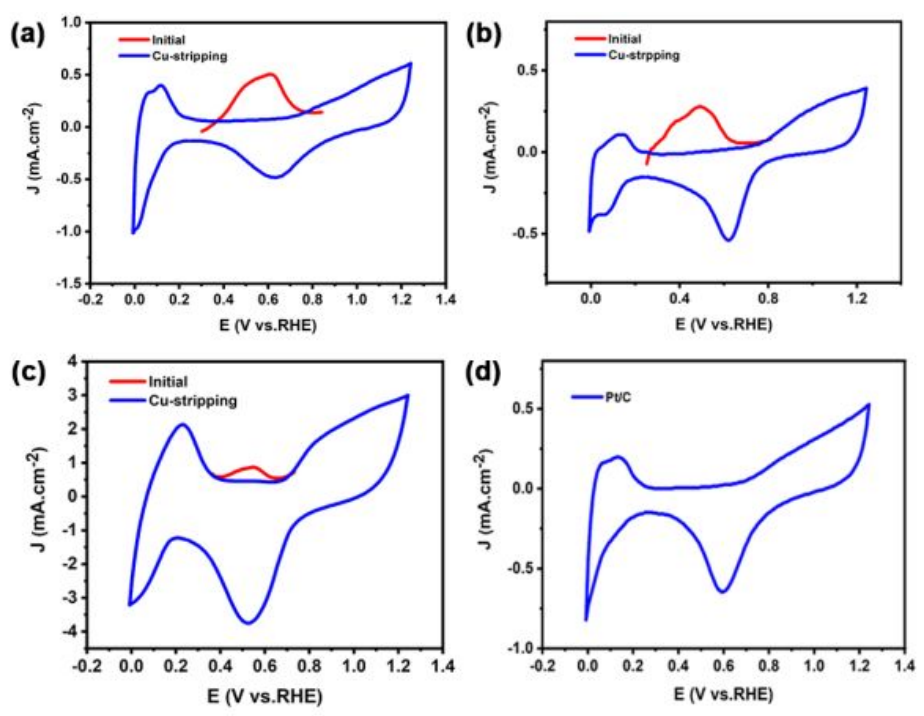

Figure S12. (a) the typical CV curves and Cu stripping voltammograms of Fe-Pd UPM, (b) Pd Metallene, (c) commercial $\mathrm{Pd} / \mathrm{C}$ in the $0.05 \mathrm{M} \mathrm{H}_{2} \mathrm{SO} 4$ solution, whereas $\mathrm{Cu}$ stripping voltammograms were conducted in the $0.05 \mathrm{M} \mathrm{H}_{2} \mathrm{SO} 4+2 \mathrm{mM} \mathrm{CuSO}_{4}$ at the scan rate of $20 \mathrm{mV} \mathrm{s}$ 1 , and (d) the $\mathrm{CV}$ curve of commercial $\mathrm{Pt} / \mathrm{C}$ in the $0.05 \mathrm{M} \mathrm{H}_{2} \mathrm{SO}_{4}$ solution.

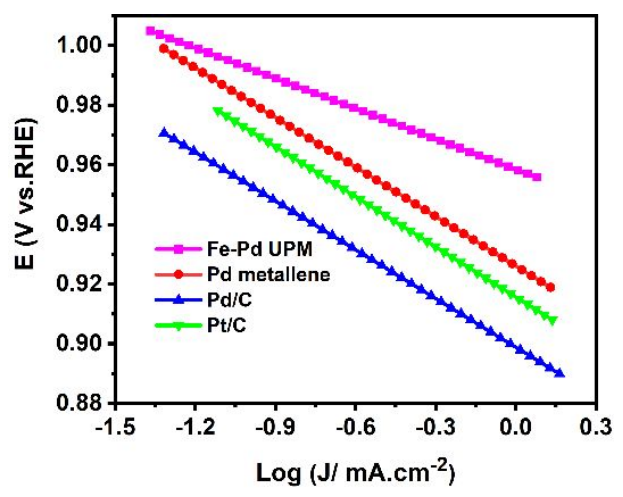

Figure S13. The Tafel plot of Fe-Pd UPM, Pd metallene, commercial Pd/C, and commercial Pt/C. 
(a)

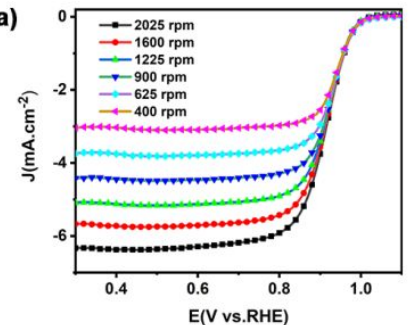

(c)

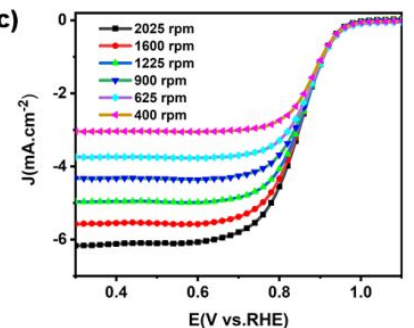

(b)

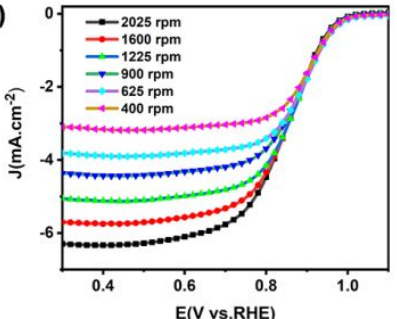

(d)

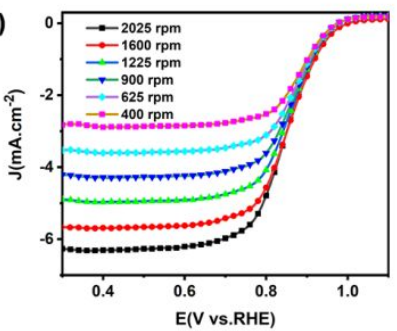

Figure S14. ORR polarization curves of (a) Fe-Pd UPM, (B) Pd metallene, (C) commercial Pd/C, and (D) commercial $\mathrm{Pt} / \mathrm{C}$ at rotation rate varying from 400 to $2025 \mathrm{rpm}$.

(a)

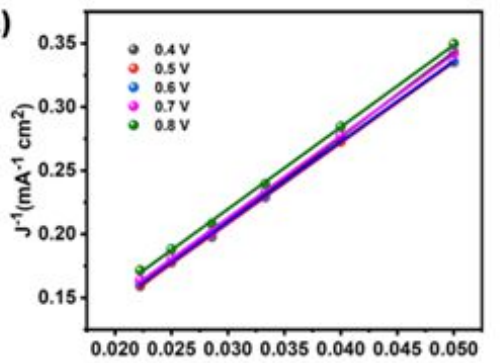

$\omega^{-1 / 2}\left(\mathrm{rpm}^{-1 / 2}\right)$

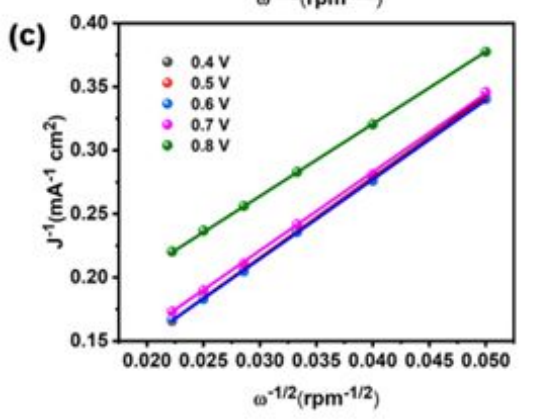

(b)

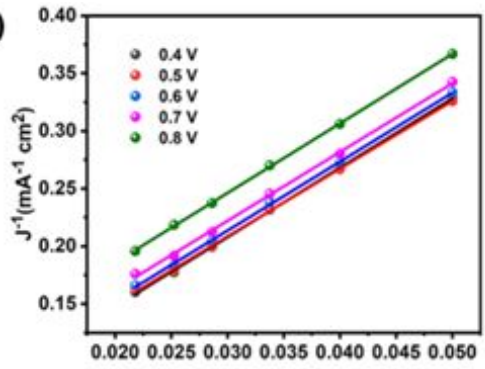

$\omega^{-1 / 2}\left(\mathrm{rpm}^{-1 / 2}\right)$

(d)

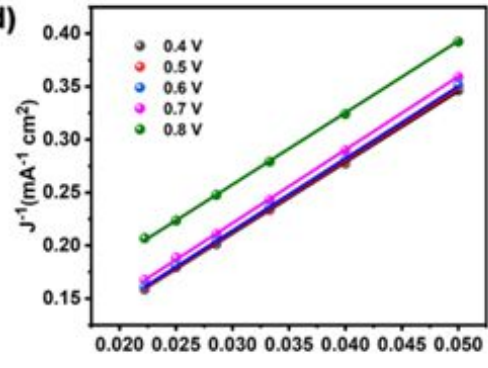

Figure S15. K-L Plots of (a) Fe-Pd UPM, (B) Pd metallene, (C) commercial Pd/C, and (D) commercial $\mathrm{Pt} / \mathrm{C}$ according to the Koutecky-Levich equation at electrode potentials ranging from 0.4 to $0.8 \mathrm{~V}$ (vs. RHE) 


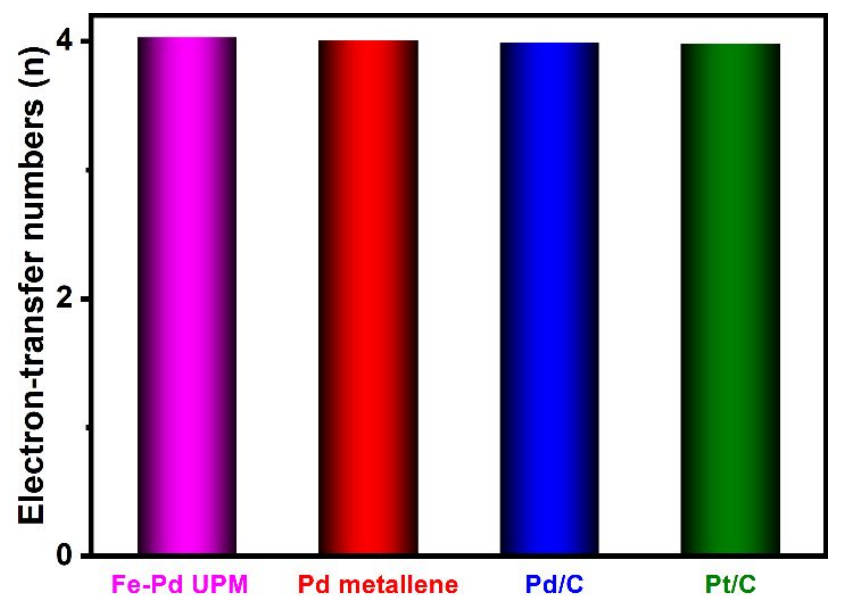

Figure S16. The electron-transfer numbers of Fe-Pd UPM, Pd metallene, commercial Pd/C, and commercial Pt/C.
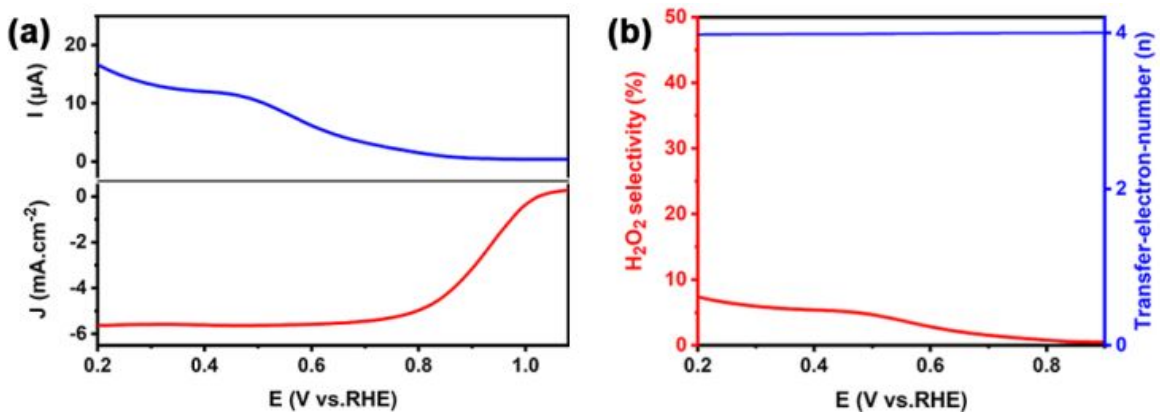

Figure S17. (a) The ORR polarization plots of Fe-Pd UPM were conducted by RRDE setup at 1600 rpm, and (b) the corresponding $\mathrm{H}_{2} \mathrm{O}_{2}$ selectivity.

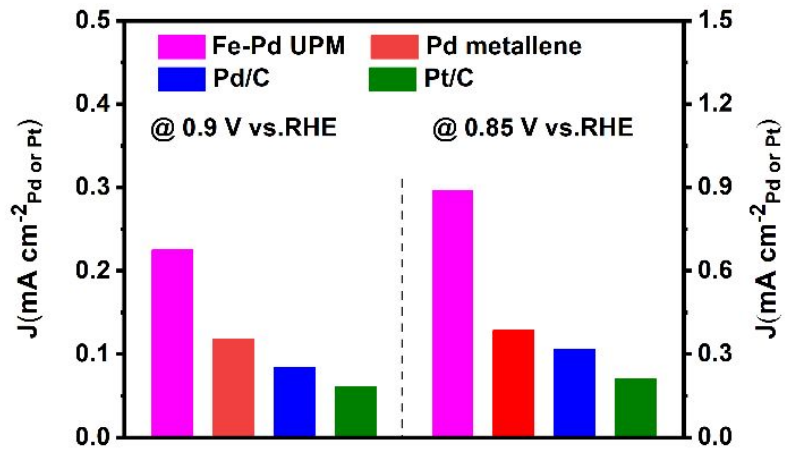

Figure S18. The specific activity of Fe-Pd UPM, Pd metallene, commercial Pd/C, and commercial $\mathrm{Pt} / \mathrm{C}$ at the $0.9 \mathrm{~V}$ and $0.85 \mathrm{~V}$ vs. RHE. 

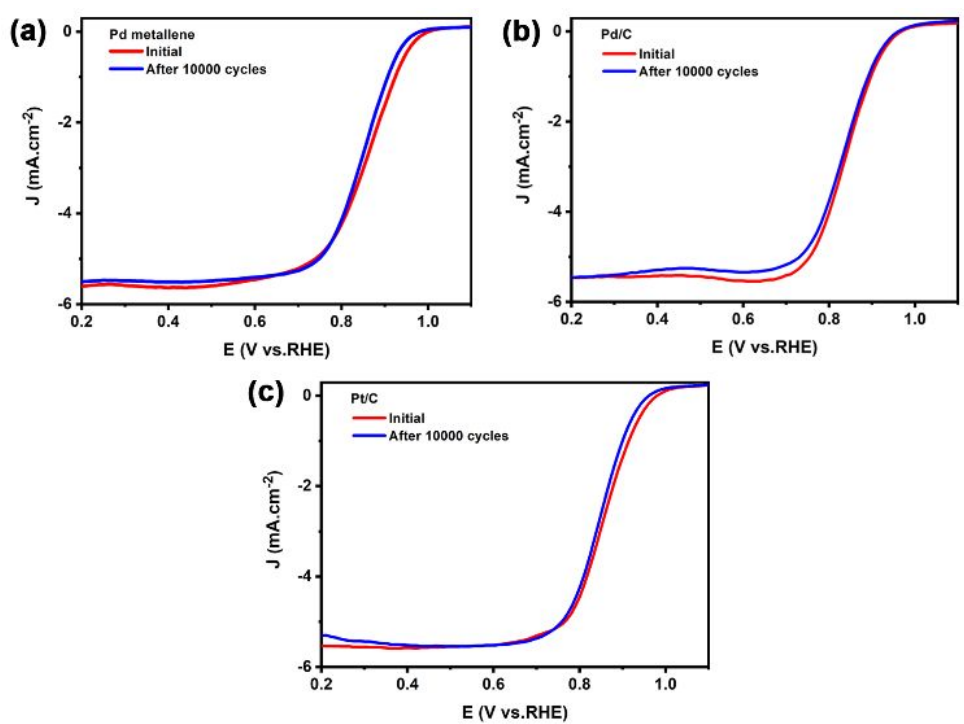

Figure S19. (a) The ORR polarization plots of (a) Pd metallene, (b) commercial Pd/C, and (c) commercial Pt/C before and after 10000 cycles.
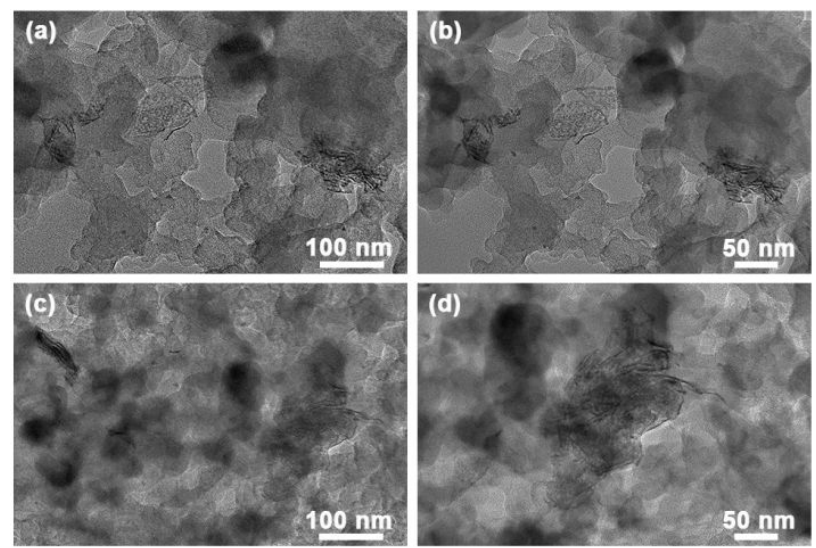

Figure S20. $(a, b)$ The TEM images of Fe-Pd UPM/C, and (c, d) corresponding images after durability test.

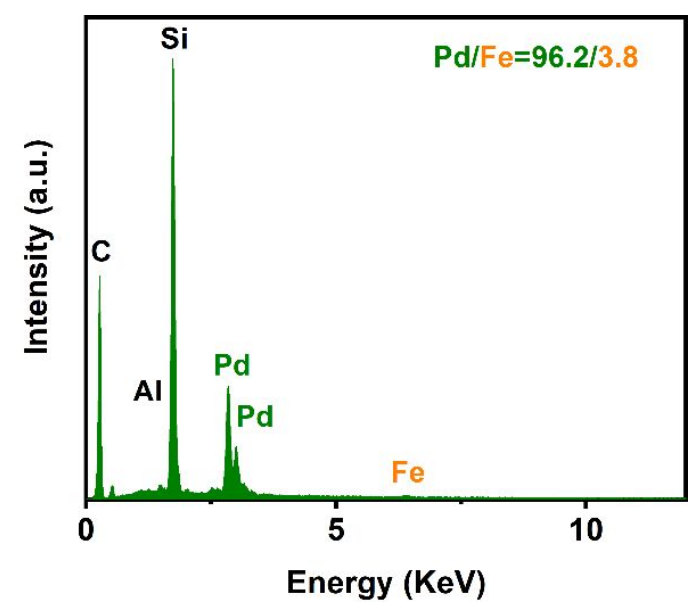

Figure S21. the EDX spectrum of Fe-Pd UPM/C after durability test. 

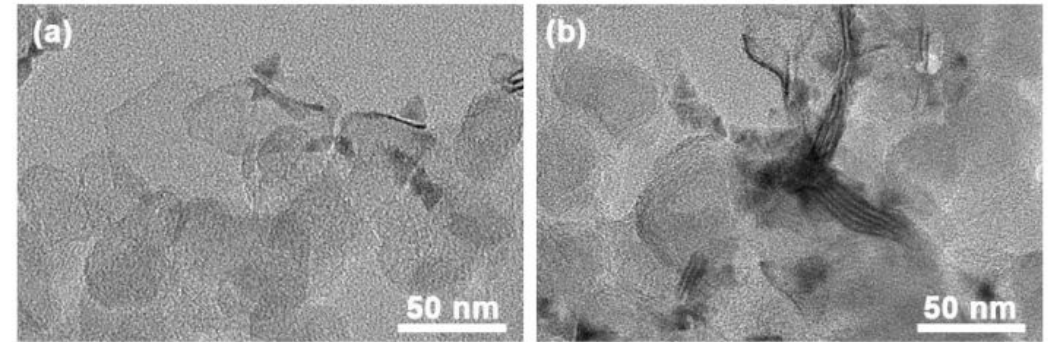

Figure S22. (a) the TEM image of Pd metallene/C, and (b) corresponding images after durability test.
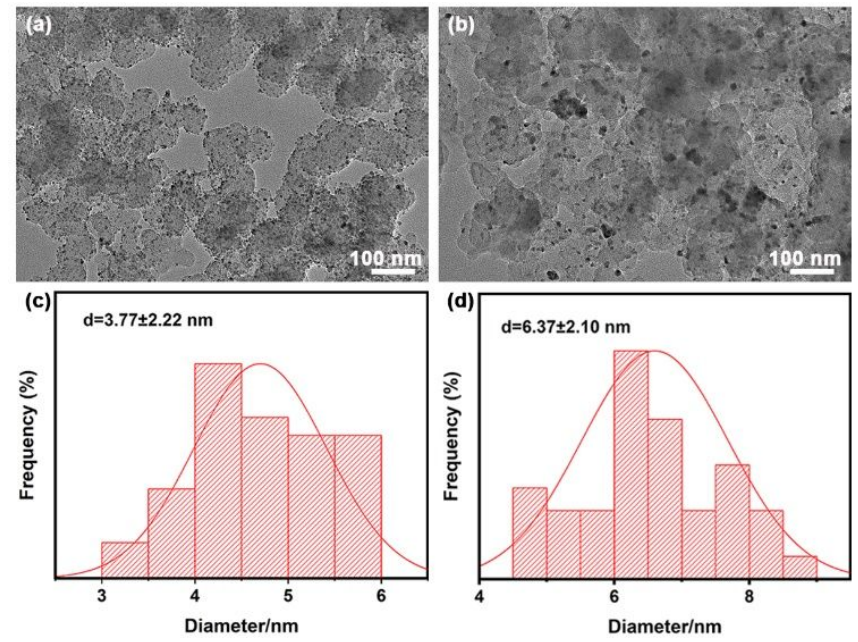

Figure S23. (a, b) The TEM image of commercial Pd/C, and (c, d) corresponding particle size profile before and after durability test.
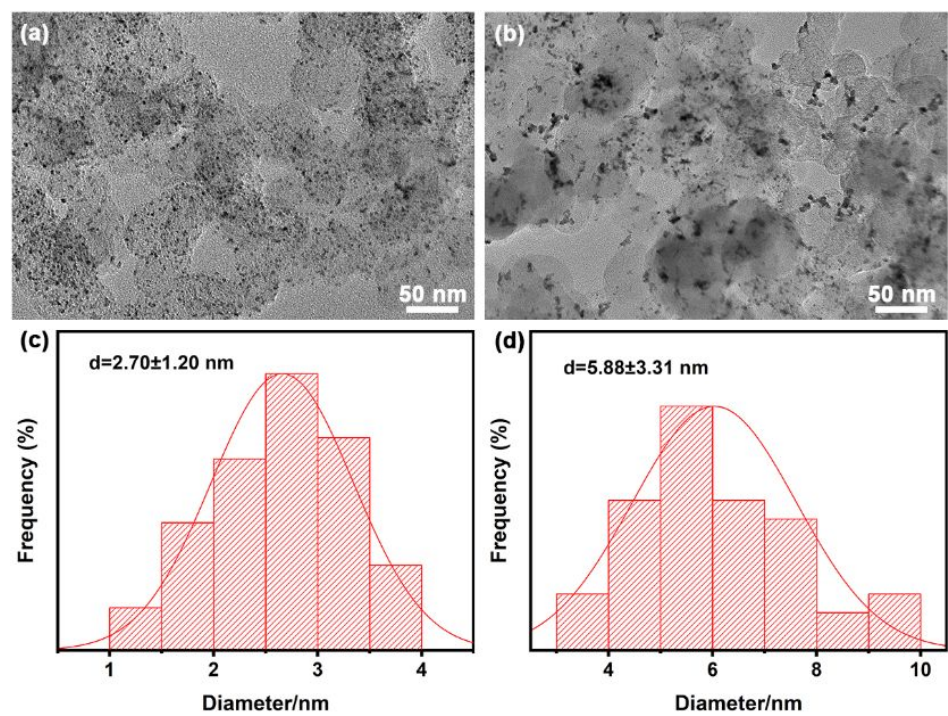

Figure S24. (a, b) The TEM image of commercial Pt/C, and (c, d) corresponding particle size profile before and after durability test. 


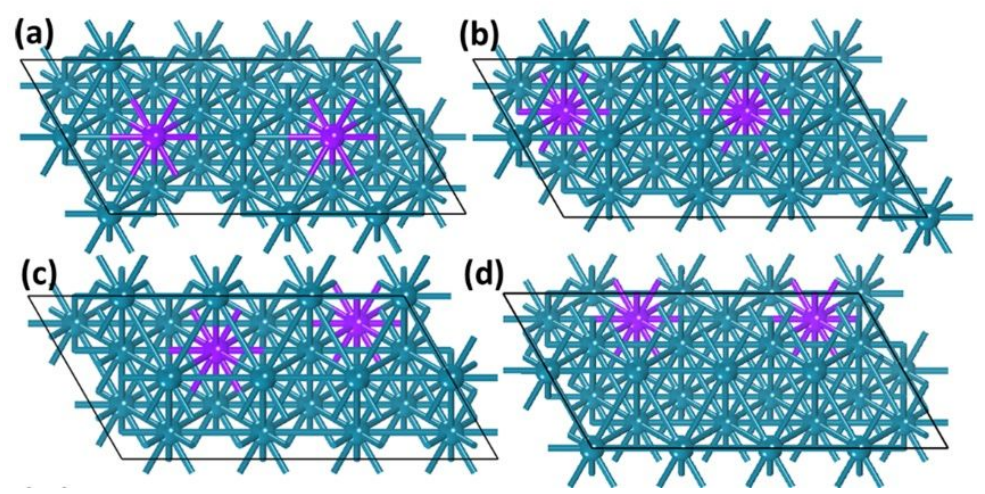

Figure S25. The topview of four structures for Fe nonneighborly dispersed on $\mathrm{Pd}(111)$, including (a) Fe at surface layer; (b) the second-layer; (c) the second-third layer; (d) the third layer.

(a)

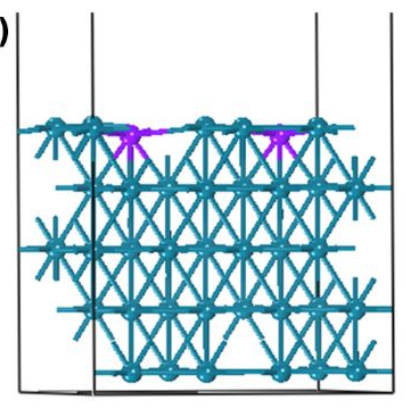

(c)

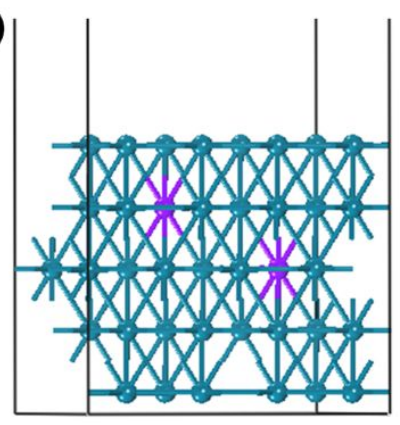

(b)

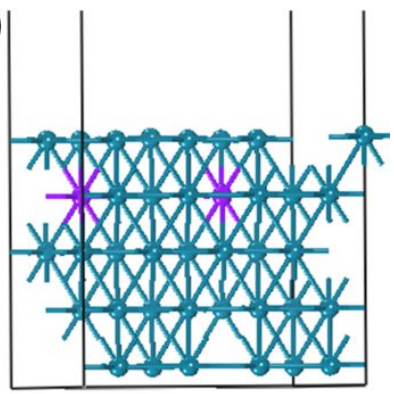

(d)

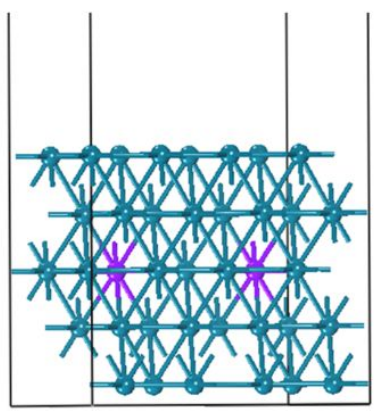

Figure S26. The sideview of four structures for Fe nonneighborly dispersed on Pd (111), including (a) Fe at surface layer; (b) the second-layer; (c) the second-third layer; (d) the third layer.
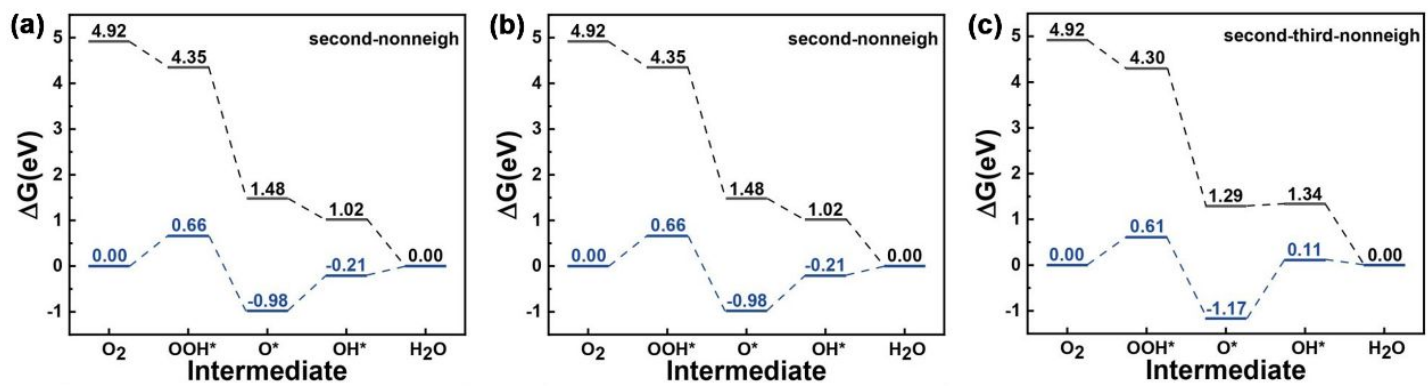

Figure S27. The free energy variation of ORR processes on Fe-dispersed on Pd (111) surface layer, second layer and second-third layer electrocatalysts. 

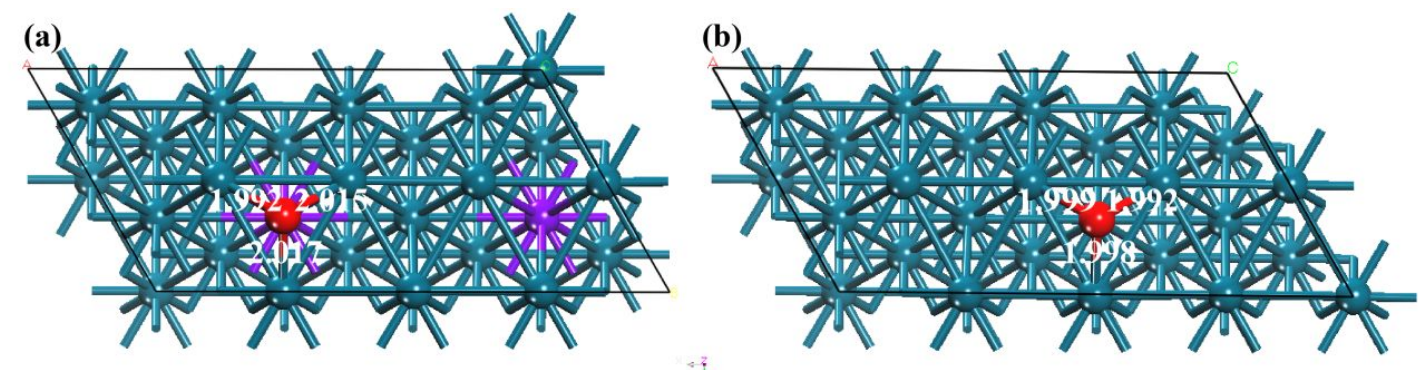

Figure S28. The bond-length of $\mathrm{O}^{*}$-intermediate for Fe-2 ${ }^{\text {nd }}$-layer $\mathrm{Pd}(111)$ (a) and pristine $\mathrm{Pd}(111)$ (b) to show the slightly longer Pd-O bond after Fe-doped in sublayer Pd(111). Blue, red and purple balls represent $\mathrm{Pd}, \mathrm{O}$ and $\mathrm{Fe}$ atoms, respectively.
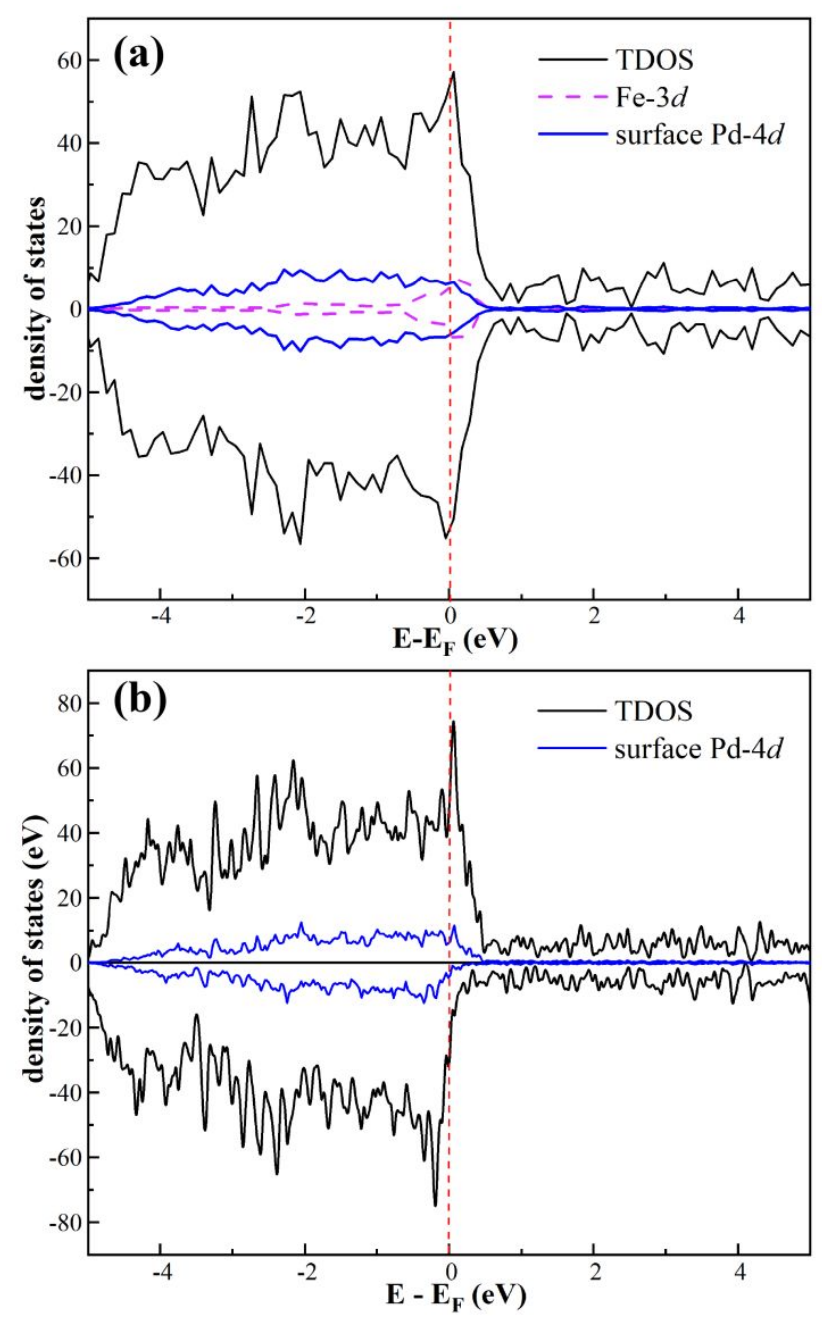

Figure S29: The calculated total DOS (TDOS) and local DOS of Fe non-neighborly dispersed into the (a) $2^{\text {nd }}$ layer Pd (111) and (b) pristine Pd (111). 
Table S1. The ORR activity of recent reported Pd-based metal catalysts in the basic condition (NA: No analysis).

\begin{tabular}{|c|c|c|c|c|c|c|c|c|c|}
\hline Catalysts & $\begin{array}{c}\text { Test } \\
\text { codition }\end{array}$ & $\begin{array}{c}\text { Half-wave } \\
\text { potential }\end{array}$ & $\begin{array}{l}\text { ECSAs } \\
\left(\mathrm{m}^{2} \mathrm{~g}^{1} \mathrm{Pd}^{-}\right.\end{array}$ & $\begin{array}{l}\text { Specific } \\
\text { Activity } \\
\left(\mathrm{mA} / \mathrm{cm}^{-2}\right)\end{array}$ & $\begin{array}{c}\text { Mass Activity } \\
\text { (A/mg) }\end{array}$ & $\begin{array}{c}\text { accelerated } \\
\text { durability tests }\end{array}$ & $\begin{array}{l}\text { Mass activity } \\
\quad \text { loss }\end{array}$ & \begin{tabular}{|c|} 
Degradation of \\
Half-wave \\
potential
\end{tabular} & Reference \\
\hline Fe-Pd UPM & $\begin{array}{l}\text { 0.1 M KOH } \\
\text { Scan rate: } \\
10 \mathrm{mV} \cdot \mathrm{s}^{-1}\end{array}$ & $\begin{array}{l}0.914 \text { (V vs. } \\
\text { RHE) }\end{array}$ & 81.95 & $\begin{array}{l}0.225(0.9 \mathrm{~V} v s . \\
\text { RHE) }\end{array}$ & $\begin{array}{l}0.736(0.9 \mathrm{~V} \\
\text { vs. RHE })\end{array}$ & $10 \mathrm{~K}$ cycles & $5.1 \%$ & No shift & This work \\
\hline $\mathbf{P d}_{3} \mathrm{~Pb}$ UPIN & $\begin{array}{c}0.1 \mathrm{M} \mathrm{KOH} \\
\text { Scan rate: } 10 \\
\mathrm{mV} \cdot \mathrm{s}^{-1}\end{array}$ & $\begin{array}{c}0.908(\mathrm{~V} v s . \\
\text { RHE) }\end{array}$ & 50.1 & $\begin{array}{c}1.18(0.9 \mathrm{~V} v s . \\
\text { RHE) }\end{array}$ & $\begin{array}{c}0.59(0.9 \mathrm{~V} v s . \\
\text { RHE) }\end{array}$ & $10 \mathrm{~K}$ cycles & $13.5 \%$ & Negligible shift & $\begin{array}{c}\text { Angew. Chem. Int. Ed. } \\
2021,60,10942 .\end{array}$ \\
\hline PdBi_8h nanoparticle & $\begin{array}{c}0.1 \mathrm{M} \mathrm{KOH} \\
\text { Scan rate: } 10 \\
\mathrm{mV} \cdot \mathrm{s}^{-1}\end{array}$ & $\begin{array}{c}\text { Shifted positively } \\
\text { by } 80 \mathrm{mV} \\
\text { relative to } \mathrm{Pt} / \mathrm{C}\end{array}$ & NA & $\begin{array}{c}2.07 \text { (0.85 V vs. } \\
\text { RHE) }\end{array}$ & $\begin{array}{c}0.182(0.85 \mathrm{~V} \\
v s . \text { RHE) }\end{array}$ & NA & NA & NA & $\begin{array}{l}\text { ACS Catal. 2021, } 11, \\
800-808\end{array}$ \\
\hline $\begin{array}{c}\mathrm{Pd}_{4} \mathrm{Au} \\
\text { hollow nanochains }\end{array}$ & \begin{tabular}{|c|}
$0.1 \mathrm{M}$ \\
KOH Scan \\
rate: 10 \\
$\mathrm{mV} \cdot \mathrm{s}^{-1}$ \\
\end{tabular} & $\begin{array}{l}0.874(\mathrm{~V} v s . \\
\text { RHE) }\end{array}$ & $5.99\left(\mathrm{~cm}^{-2}\right)$ & $\begin{array}{l}0.137(0.85 \mathrm{~V} \\
\quad v s . \text { RHE) }\end{array}$ & $\begin{array}{c}0.287(0.85 \mathrm{~V} \\
v s . \text { RHE) }\end{array}$ & NA & NA & NA & $\begin{array}{c}\text { Adv. Energy Mater. 2020, } \\
10,1904072 .\end{array}$ \\
\hline $\begin{array}{c}\mathrm{Pd}_{20} \mathrm{Te}_{7} \text { hexagonal } \\
\text { nanoplates }\end{array}$ & $\begin{array}{c}0.1 \mathrm{M} \mathrm{KOH} \\
\text { Scan rate: } 10 \\
\mathrm{mV} \cdot \mathrm{s}^{-1}\end{array}$ & 0.90 (V vs. RHE) & NA & NA & $\begin{array}{l}0.3 \text { (0.9 V vs. } \\
\text { RHE) }\end{array}$ & $20 \mathrm{~K}$ cycles & $16 \%$ & No Obvious & $\begin{array}{c}\text { Sci. Adv. 2020, 6, eaba } \\
9731 .\end{array}$ \\
\hline PdCu nanoparticle & $\begin{array}{c}1 \mathrm{M} \mathrm{KOH} \\
\begin{array}{c}\text { Scan rate: } 5 \\
\mathrm{mV} \cdot \mathrm{s}^{-1}\end{array}\end{array}$ & $\begin{array}{l}0.886(\mathrm{~V} v s . \\
\text { RHE) }\end{array}$ & 54 & $\begin{array}{c}2.3 \text { ( } 0.9 \mathrm{~V} \text { vs. } \\
\text { RHE) }\end{array}$ & $\begin{array}{c}0.13 \text { (0.9 V vs. } \\
\text { RHE) }\end{array}$ & $100 \mathrm{~K}$ cycles & $43 \%$ & $18 \mathrm{mV}$ & $\begin{array}{c}\text { J. Am. Chem. Soc. 2020, } \\
\text { 142, 3980-3988. }\end{array}$ \\
\hline $\begin{array}{c}\text { PdZn } \\
\text { core-shell }\end{array}$ & $\begin{array}{c}1 \mathrm{M} \mathrm{KOH} \\
\text { Scan rate: } 10 \\
\mathrm{mV} \cdot \mathrm{s}^{-1}\end{array}$ & 0.82 (V vs. RHE) & 90.22 & $\begin{array}{c}0.048(0.9 \mathrm{~V} v s . \\
\text { RHE) }\end{array}$ & $\begin{array}{c}0.044(0.9 \mathrm{~V} \\
v s . \text { RHE) }\end{array}$ & $2.5 \mathrm{~K}$ cycles & NA & $12 \mathrm{mV}$ & $\begin{array}{c}\text { J. Catal. 2020, 382, } 181- \\
191 .\end{array}$ \\
\hline $\begin{array}{l}\text { Pd@PdFe core-shell } \\
\text { icosahedra }\end{array}$ & $\begin{array}{c}0.1 \mathrm{M} \mathrm{KOH} \\
\text { Scan rate: } 10 \\
\mathrm{mV} \cdot \mathrm{s}^{-1}\end{array}$ & 0.92 (V vs. RHE) & 19.8 & $\begin{array}{c}1.56(0.9 \mathrm{~V} v s . \\
\text { RHE) }\end{array}$ & $\begin{array}{c}0.31(0.9 \mathrm{~V} v s . \\
\text { RHE) }\end{array}$ & $20 \mathrm{~K}$ cycles & $15 \%$ & No Obvious & $\begin{array}{l}\text { Nano Lett. 2020, 20, 1403- } \\
1409 .\end{array}$ \\
\hline $\mathbf{P d}_{31} \mathbf{B i}_{12}$ nanoparticles & $\begin{array}{c}0.1 \mathrm{M} \mathrm{KOH} \\
\text { Scan rate: } 20 \\
\mathrm{mV} \cdot \mathrm{s}^{-1}\end{array}$ & 0.92 (V vs. RHE) & NA & $\begin{array}{c}2.42 \pm 0.20 \\
(0.9 \mathrm{~V} v s . \mathrm{RHE})\end{array}$ & $\begin{array}{c}0.95 \pm 0.18 \\
(0.9 \mathrm{~V} v s . \\
\text { RHE })\end{array}$ & $10 \mathrm{~K}$ cycles & $40 \%$ & NA & $\begin{array}{c}\text { ACS Energy Lett. 2020, } 5, \\
17-22 .\end{array}$ \\
\hline $\begin{array}{c}\text { Ordered Intermetallic } \\
\mathbf{P d}_{3} \mathbf{B i}\end{array}$ & $\begin{array}{c}0.1 \mathrm{M} \mathrm{KOH} \\
\text { Scan rate: } 20 \\
\mathrm{mV} \cdot \mathrm{s}^{-1}\end{array}$ & NA & NA & $\begin{array}{c}2.3 \pm 0.19(0.9 \\
\text { V vs. RHE) }\end{array}$ & $\begin{array}{c}1.2 \pm 0.08 \\
(0.9 \mathrm{~V} v s . \\
\text { RHE) }\end{array}$ & $10 \mathrm{~K}$ cycles & $31 \%$ & NA & $\begin{array}{l}\text { ACS Nano 2019, 13, } \\
\text { 10818-10825. }\end{array}$ \\
\hline $\begin{array}{c}\text { Ordered Au } \\
\text { incorporated PdZn }\end{array}$ & $\begin{array}{c}0.1 \mathrm{M} \mathrm{KOH} \\
\text { Scan rate: } 5 \\
\mathrm{mV} \cdot \mathrm{s}^{-1}\end{array}$ & $\begin{array}{c}\text { Shifted positively } \\
\text { by } 50 \mathrm{mV} \\
\text { relative to } \mathrm{Pt} / \mathrm{C}\end{array}$ & NA & NA & $\begin{array}{c}0.105(0.9 \mathrm{~V} \\
v s . \text { RHE })\end{array}$ & $30 \mathrm{~K}$ cycles & $9.5 \%$ & $6 \mathrm{mV}$ & $\begin{array}{c}\text { ACS Nano } 2019,13,5968- \\
5974 .\end{array}$ \\
\hline $\begin{array}{l}\mathrm{Pd}_{3} \mathrm{~Pb} / \mathrm{Pd} \text { tetragonal } \\
\text { nanosheets }\end{array}$ & $\begin{array}{c}0.1 \mathrm{M} \\
\mathrm{KOH} \mathrm{Scan} \\
\text { rate: } 10 \\
\mathrm{mV} \cdot \mathrm{s}^{-1}\end{array}$ & NA & 43.7 & $\begin{array}{c}1.31 \text { (0.9 V vs. } \\
\text { RHE) }\end{array}$ & $\begin{array}{c}0.57(0.9 \mathrm{~V} v s . \\
\text { RHE) }\end{array}$ & $20 \mathrm{~K}$ cycles & $8.1 \%$ & NA & $\begin{array}{c}\text { Nano Lett. 2019, 19, } 2, \\
\quad 1336-1342 .\end{array}$ \\
\hline PdMo bimetallene & \begin{tabular}{|c|}
$0.1 \mathrm{M}$ \\
KOH Scan \\
rate: 20 \\
$\mathrm{mV} \cdot \mathrm{s}^{-1}$
\end{tabular} & 0.95 & $138.7 \pm 9.1$ & $1 \begin{array}{c}11.64 \pm 0.40 \\
(0.9 \mathrm{~V} v s . \text { RHE })\end{array}$ & $\begin{array}{c}16.37 \pm 0.60 \\
(0.9 \mathrm{~V} v s . \\
\text { RHE) }\end{array}$ & $30 \mathrm{~K}$ cycles & around $32 \%$ & NA & Nature 2019, 574, 81-85. \\
\hline $\begin{array}{l}\text { PdCuNi alloy } \\
\text { nanocrystals }\end{array}$ & $\begin{array}{c}0.1 \mathrm{M} \mathrm{NaOH} \\
\text { Scan rate: } 10 \\
\mathrm{mV} \cdot \mathrm{s}^{-1}\end{array}$ & 0.90 (V vs. RHE) & NA & NA & $\begin{array}{c}0.45(0.9 \mathrm{~V} v s . \\
\text { RHE) }\end{array}$ & NA & NA & NA & $\begin{array}{c}\text { Adv. Funct. Mater. } 2018, \\
28,1707219 .\end{array}$ \\
\hline $\mathrm{Pd}_{6} \mathrm{Ni}$ icosahedra & $\begin{array}{c}0.1 \mathrm{M} \mathrm{KOH} \\
\text { Scan rate: } 10 \\
\mathrm{mV} \cdot \mathrm{s}^{-1}\end{array}$ & NA & 47.5 & $\begin{array}{c}0.66(0.9 \mathrm{~V} v s . \\
\text { RHE) }\end{array}$ & $\begin{array}{c}0.22(0.9 \mathrm{~V} v s . \\
\text { RHE) }\end{array}$ & $10 \mathrm{~K}$ cycles & $5.6 \%$ & No Obvious & $\begin{array}{l}\text { Sci. Adv. 2018, } 4 \\
\text { eaap } 8817 .\end{array}$ \\
\hline Dentritic $\mathrm{Pd}_{59} \mathrm{Cu}_{30} \mathrm{Co}_{11}$ & $\begin{array}{c}0.1 \mathrm{M} \mathrm{KOH} \\
\text { Scan rate: } 10 \\
\mathrm{mV} \cdot \mathrm{s}^{-1}\end{array}$ & 0.91 (V vs. RHE) & 46.3 & $\begin{array}{l}0.90(0.9 \mathrm{~V} v s . \\
\text { RHE) }\end{array}$ & $\begin{array}{c}0.38(0.9 \mathrm{~V} v s . \\
\text { RHE) }\end{array}$ & $15 \mathrm{~K}$ cycles & $31.6 \%$ & NA & $\begin{array}{c}\text { Nat. Commun. 2018, } 9 \text {, } \\
3702 .\end{array}$ \\
\hline $\mathbf{P d}_{3} \mathbf{P b}$ Tripods & $\begin{array}{c}1 \mathrm{M} \mathrm{KOH} \\
\text { Scan rate: } 10 \\
\mathrm{mV} \cdot \mathrm{s}^{-1}\end{array}$ & NA & 31.9 & $\begin{array}{c}1.76(0.9 \mathrm{~V} v s . \\
\text { RHE) }\end{array}$ & $\begin{array}{c}0.56(0.9 \mathrm{~V} v s . \\
\text { RHE })\end{array}$ & $20 \mathrm{~K}$ cycles & $16.6 \%$ & NA & Chem 2018, 4, 359-371. \\
\hline Au-NWs@Pd@PEI & $\begin{array}{c}0.1 \mathrm{M} \mathrm{KOH} \\
\begin{array}{c}\text { Scan rate: } 5 \\
\mathrm{mV} \cdot \mathrm{s}^{-1}\end{array}\end{array}$ & 0.90 (V vs. RHE) & 74 & NA & $\begin{array}{c}0.29(0.9 \mathrm{~V} v s . \\
\text { RHE) }\end{array}$ & $10 \mathrm{~K}$ cycles & $69.7 \%$ & $38 \mathrm{mV}$ & $\begin{array}{c}\text { ACS Catal. 2018, 8, } 11287- \\
11295 .\end{array}$ \\
\hline $\begin{array}{c}\text { Ordered PdCuCo } \\
\text { NPs/C }\end{array}$ & $\begin{array}{c}1 \mathrm{M} \mathrm{NaOH} \\
\text { Scan rate: } 100 \\
\mathrm{mV} \cdot \mathrm{s}^{-1}\end{array}$ & 0.872 (V vs. RHE) & NA & NA & $\begin{array}{c}0.13(0.9 \mathrm{~V} v s . \\
\text { RHE) }\end{array}$ & $10 \mathrm{~K}$ cycles & $3.9 \%$ & No Obvious & $\begin{array}{l}\text { Angew. Chem. Int. Ed. } \\
2016,128,9176-9181 .\end{array}$ \\
\hline
\end{tabular}

